\title{
Sífilis congênita: caracterização epidemiológica no estado do Piauí, Brasil
}

\author{
Congenital syphilis: epidemiological characterization in the state of Piauí, Brazil \\ Sífilis congénita: caracterización epidemiológica en el estado del Piauí, Brasil
}

Ivete Rodrigues Nunes ${ }^{1}$, Maria Dalila Rodrigues Ribeiro ${ }^{1}$, Kécya Patricia Costa Macêdo ${ }^{1}$ Jéssica Larissa Sousa Vaz ${ }^{2}$, Soliane Cristina Rodrigues Costa ${ }^{2}$, Matheus Costa Silva ${ }^{1}$, Bianca de Sousa Leal $^{1}$, Roseane Mara Cardoso Lima Verde ${ }^{3}$, Evaldo Hipólito de Oliveira ${ }^{2 *}$.

\section{RESUMO}

Objetivo: Estabelecer o predomínio da sífilis congênita (SC) caracterizando o perfil epidemiológico e fatores associados à transmissão no Piauí. Métodos: Pesquisa descritiva epidemiológica, realizada através do Sistema de Informação de Agravos de Notificação - SINAN para estudar o perfil epidemiológico da SC no Piauí de 2013 a 2017. As informações foram coletadas no mês de julho de 2018, entre 2013 a 2017. Foram coletados no SINAN ( $N=1624)$. Resultados: Observou-se que a maior ocorrência de casos aconteceu a partir de 2015 (201,36\%). Em 2013 o percentual de sífilis congênita foi de 3,17 por nascidos vivos, onde houve um aumento substancial para 8,63 casos 2015 e 9,28 casos em 2016. Conclusão: A pesquisa mostra que ainda há muitos casos da doença no estado do Piauí, com isso o estudo visou contribuir para uma reflexão acerca de maiores conhecimentos sobre a sífilis congênita evidenciando-se a necessidade da presença de mais estudos nesse sentido. Todavia, é com assertividade que este trabalho irá facilitar futuras propostas que visem conhecer e estabelecer o predomínio da SC de forma que caracterize o perfil epidemiológico e fatores associados à transmissão no Piauí.

Palavras-chave: Epidemiologia, Caracterização, Sífilis congênita.

\begin{abstract}
Objective: To establish the prevalence of congenital syphilis (CS) characterizing the epidemiological profile and factors associated with transmission in Piauí. Methods: Descriptive epidemiological research carried out through the SINAN Information System to study the epidemiological profile of CS in Piauí from 2013 to 2017. The information was collected in July 2018, between 2013 and 2017 . There were collected in SINAN $(N=$ 1624). Results: It was observed that the highest occurrence of cases occurred from 2015 (201.36\%). In 2013 the percentage of congenital syphilis was 3.17 per live birth, where there was a substantial increase to 8.63 cases in 2015 and 9.28 cases in 2016. Conclusion: The research shows that there are still many cases of the disease in the state of Piauí, so the study aimed to contribute to a reflection about greater knowledge about congenital syphilis evidencing the need for more studies in this sense. However, it is with assertiveness that this work will facilitate future proposals that seek to know and establish the prevalence of SC in a way that characterizes the epidemiological profile and factors associated with transmission in Piauí.
\end{abstract}

Keyword: Epidemiology, Characterization, Congenital syphilis.

${ }^{1}$ Centro Universitário Santo Agostinho. Teresina - PI.

2Universidade Federal do Piauí. Centro de Ciências da Saúde. Teresina - PI.

*E-mail: evaldohipolito@gmail.com

${ }^{3}$ Programa de Pós-Graduação em Engenharia Biomédica - Universidade Brasil. São Paulo - SP. 


\section{RESUMEN}

Objetivo: Establecer el predominio de la sífilis congénita (SC) caracterizando el perfil epidemiológico y factores asociados a la transmisión en Piauí. Métodos: Investigación descriptiva epidemiológica, realizada a través del Sistema de Información de Agravios de Notificación - SINAN para estudiar el perfil epidemiológico de la SC en Piauí de 2013 a 2017. Las informaciones fueron recolectadas en el mes de julio de 2018, entre 2013 a 2017. Fueron recolectados en el SINAN ( $=1624)$. Resultados: Se observó que la mayor ocurrencia de casos ocurrió a partir de 2015 (201,36\%). En 2013 el porcentaje de sífilis congénita fue de 3,17 por nacidos vivos, donde hubo un aumento sustancial para 8,63 casos 2015 y 9,28 casos en 2016. Conclusión: La investigación muestra que aún hay muchos casos de la enfermedad en el estado de la enfermedad Piauí, con ello el estudio pretendió contribuir a una reflexión acerca de mayores conocimientos sobre la sífilis congénita evidenciándose la necesidad de la presencia de más estudios en ese sentido. Sin embargo, es con asertividad que este trabajo facilitará futuras propuestas que visen conocer y establecer el predominio de la SC de forma que caracterice el perfil epidemiológico y factores asociados a la transmisión en Piauí.

Palabras clave: Epidemiología, Caracterización, Sífilis congénita.

\section{INTRODUÇÃO}

A sífilis é uma patologia crônica, de grande relevância para a Saúde Pública, destacando-se entre as infecções sexualmente transmissíveis (IST's) mais notificada no Brasil. A sua transmissão pode ser dada através do ato sexual, da placenta ou da transfusão sanguínea. Uma vez ocorrido o meio de transmissão por via placentária, resulta-se em uma doença denominada sífilis congênita (SC). Por sua vez, esta é uma infecção causada ao feto pelo Treponema pallidum. É uma patologia de múltiplos sistemas que não detectada durante a gestação ou não sendo tratada precocemente, é capaz de provocar efeitos nocivos à saúde. O seu avanço ocorre através de etapas, podendo manifestar sintomas ou não, como consequência pode acometer qualquer órgão do corpo humano (CASAGRANDE A, et al., 2017).

A grande maioria dos recém-nascidos que contraem a doença é infectada no útero e/ou ao nascer, resultando-se em inúmeras complicações das quais podemos citar, distúrbios dermatológicos, neurológicos, ósseos e cardiovasculares. A sífilis apresenta fases distintas (Fase primaria, secundaria e terciaria) com sintomas específicos, na fase primaria por exemplo, que ocorre assim que há o contato com a bactéria treponema pallidum, formam-se feridas normalmente indolores nas genitais, na fase secundaria que ocorre cerca de 2 a 8 semanas após as primeiras feridas se formarem, nessa fase o paciente pode apresentar exantema por todo o corpo, ínguas, coceira, pode ainda apresentar dores musculares e febre, e na fase terciaria, esta é a mais difícil de ser tratada, pois compromete o sistema nervoso central, o sistema cardiovascular com inflamação na aorta e apresenta também lesões nos ossos (MISNITÉRIO DA SAÚDE, 2006).

No período gravídico-puerperal pode-se ocasionar vários efeitos nocivos, tais como: o abortamento espontâneo, morte fetal e neonatal, prematuridade e entre outros danos à saúde do recém-nascido, como os efeitos psicológicos e sociais (SOUZA BC e SANTANA LS, 2013).

A SC possui diagnóstico simples, realizado através de um teste sorológico chamado Venereal Disease Research Laboratory (VDRL), apesar disso sua prevalência ainda é preocupante. $O$ tratamento deve ser realizado nos três primeiros meses de gestação, neste período costuma-se evitar a infecção do feto. $O$ Ministério da Saúde indica a penicilina G benzatina, para o tratamento tanto da gestante como do concepto, vale lembrar que o parceiro deve ser igualmente tratado (CARVALHO IS e BRITO RS, 2014).

A notificação e observação desse agravo são indispensáveis para o acompanhamento da contaminação vertical (placentária), haja vista que no ano de 2011 no Brasil, chegaram a ser notificados 3,3 casos por 1000 nascidos vivos. Em relação a esse cenário o Plano Operacional tem como finalidade a diminuição da contaminação Vertical da Sífilis, tratando-a como um evento sentinela para vigilância na assistência da Atenção Básica em Saúde (ARAÚJO CL, et al., 2012). 
Diante do exposto, observa-se a relevância deste trabalho para o enriquecimento acadêmico e científico sobre esta temática, que ainda existem grandes lacunas a serem preenchidas. Dessa forma tratou-se como objetivo principal, o estabelecimento do predomínio da SC caracterizando o perfil epidemiológico e fatores associados à transmissão no Piauí.

\section{MÉTODOS}

Pesquisa descritiva epidemiológica sobre a população de estudo que constituiu as ocorrências confirmadas notificadas de SC ocorridas em mães residentes no Piauí entre os anos de 2013 a 2017 ( $\mathrm{N}=1624)$. Utilizou-se como critério de exclusão: as ocorrências não residentes no município e as notificações duplicadas. As informações foram coletadas no período do mês de julho do ano de 2018, através de uma plataforma de informações, disponibilizada pelo Departamento de Informática do SUS (DATASUS), denominada de Sistema de Informação de Agravos de Notificação (SINAN). A coleta das ocorrências identificadas teve as seguintes variáveis: ano de ocorrência, município de residência, zona de residência, faixa etária, sexo, grau de instrução da mãe, realização de pré-natal, sífilis materna, tratamento do parceiro, classificação final e evolução. A análise das informações coletadas aconteceu por meio das apurações de frequências absolutas e percentuais e organização em tabelas e gráficos utilizando o software Microsoft Excel® 2013. Para melhor análise da disseminação dos casos pelo estado, foi produzido um mapa utilizandose o programa Tab para Windows - TabWin, versão 4.14.

O cálculo da porcentagem de casos da SC foi obtido dividindo-se o número dos casos em determinado período, pela quantidade de nascidos vivos no mesmo ano e multiplicando-se o resultado por mil ( $\mathrm{N}^{\circ}$ casos $\mathrm{N} N$ nascidos) $=N \times 1.000$. Dessa forma tem-se o total de ocorrências de SC a cada 1000 nascidos vivos. Enquanto o nível de mortalidade infantil é obtido através da divisão da quantidade de óbitos por SC pela quantidade de nascidos vivos no mesmo ano, multiplicando o resultado por dez mil (№ óbitos/№ vivos) $=\mathrm{N} \mathrm{x}$ 10.000. O valor é interpretado como o número de óbitos por sífilis congênita a cada 10.000 nascidos vivos. $O$ DATASUS disponibiliza o relatório sobre a quantidade de nascidos vivos no perfil fornecido pelo Sistema de Informações sobre Nascidos Vivos (SINASC).

\section{RESULTADOS}

Conforme o SINAN, entre os anos de 2013 a 2017, notificou-se 1.624 evidências de SC no estado do Piauí, representando $1,45 \%$ do total de casos no país e $5 \%$ dos casos da região Nordeste, no mesmo período. No Gráfico 1 nota-se uma elevação considerável no total de casos a partir de 2015, mantendo-se relativamente constante nos dois anos seguintes, desse modo, entre 2013 e 2017 houve um acréscimo de 201,36\% no número de notificações. Em 2013 a taxa de contaminação por SC foi de 3,17 casos por 1000 nascidos vivos, sofrendo um aumento substancial para 8,63 casos/1000 nascidos vivos em 2015 e 9,28 casos em 2016.

Gráfico 1 - Casos confirmados de sífilis congênita notificados no Piauí segundo o ano de notificação, de 2013 a 2017.

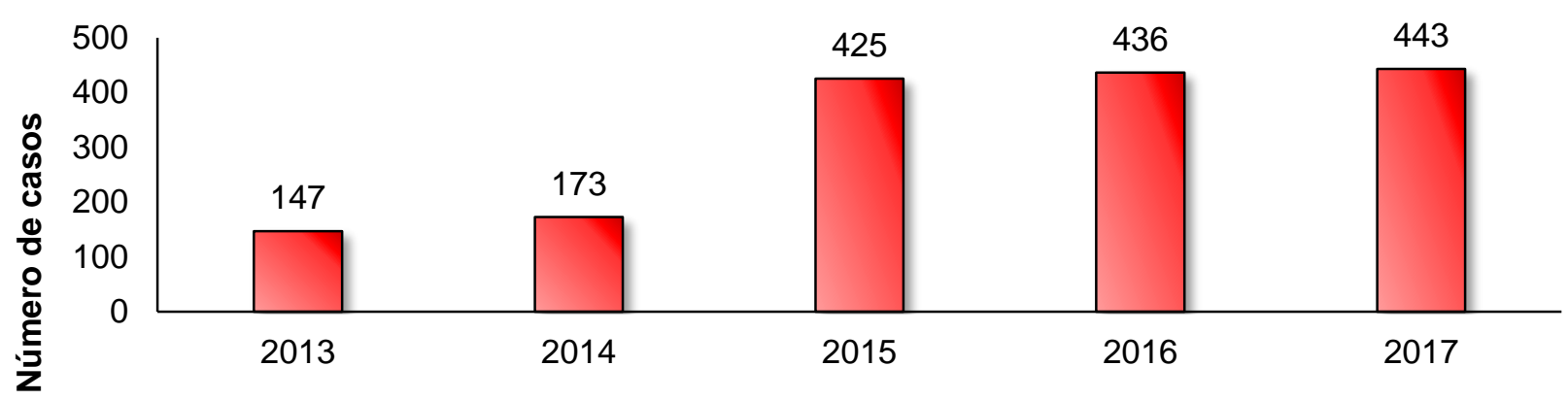

Ano de notificação

Fonte: Nunes IR, et al., 2020. Baseado em: Ministério da Saúde/SVS - Sistema de Informação de Agravos de Notificação - Sinan Net, 2018. 
Conforme ilustrado no Figura 1, dentre os 221 municípios piauienses, 137 foram citados como municípios de residência. A capital do Piauí concentrou a maioria dos casos, 848 (52,22\%), seguida do município de Parnaíba com 133 registros $(8,18 \%)$ e de Picos, com 34 notificações $(2,09 \%)$. Considerando a zona de residência, $82,77 \%$ das ocorrências acometeram indivíduos da zona urbana do estado, $15,51 \%$ pessoas residentes da zona rural, $0,06 \%$ na região periurbana e em $1,66 \%$ casos a informação foi ignorada.

Figura 1 - Casos confirmados de sífilis congênita notificados no Piauí segundo o município de residência nos anos de 2013 a 2017.

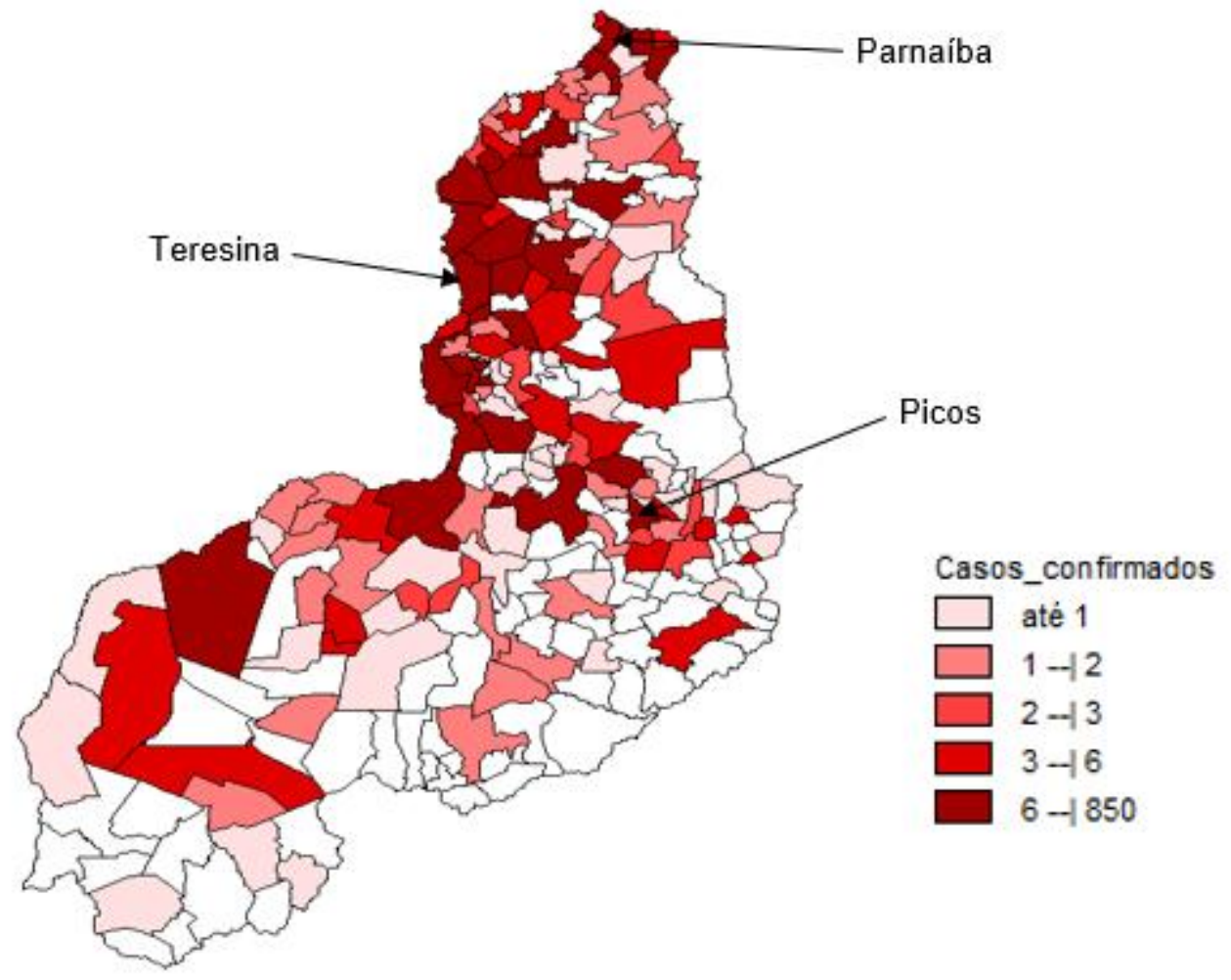

Fonte: Nunes IR, et al., 2020. Baseado em: Ministério da Saúde/SVS - Sistema de Informação de Agravos de Notificação - Sinan Net, 2018.

Totalizando as ocorrências no estado, 95,26\% (1549 casos) foram diagnosticados em bebês com até seis dias de vida, 1,84\% (30 casos) em bebês com 7 a 27 dias de nascimento e 2,83\% (46 casos) em crianças com um ano de idade (12 a 23 meses).

Evidencia-se que não foram observadas diferenças de distribuição relevantes conforme o sexo, ocorrendo 811 casos $(49,84 \%)$ entre crianças do sexo masculino, $756(46,55 \%)$ entre crianças do sexo feminino e 59 $(3,63 \%)$ registros com informação ignorada.

No tocante a escolaridade materna, evidenciou-se que o maior número $29,12 \%$, não concluiu as séries básicas do ensino fundamental ( $5^{\mathrm{a}}$ à $8^{\mathrm{a}}$ série), seguida de $17,80 \%$ com o ensino médio incompleto. Além disso, notou-se que num total de $13,05 \%$ das ocorrências essa informação foi ignorada ou classificada como não se aplica.

Os menores números foram registrados entre as mães que não cursaram, ou não concluíram o ensino superior e entre as mães consideradas analfabetas.

Conforme a Tabela 1, a assistência do pré-natal foi realizada por 85,54\% (1391) das mães de crianças com sífilis congênita, dentre as quais $54,06 \%$ (752) receberam análise positiva para sífilis materna durante esta etapa. Ainda assim, um número significativo de mães, 44,42\% (618), foram diagnosticadas apenas no momento ou pós-parto. 
Tabela 1 - Casos confirmados de sífilis materna notificados no Piauí segundo a realização de pré-natal, 2013 a 2017.

\begin{tabular}{lcccc}
\hline \multirow{2}{*}{ Sífilis materna } & \multicolumn{3}{c}{ Realização de pré-natal } & \multirow{2}{*}{ Total } \\
\cline { 2 - 4 } & Ign/Branco & Sim & Não & \\
\hline Ign/Branco & 9 & 13 & 4 & 26 \\
Durante o pré-natal & 4 & 752 & - & 756 \\
No momento do & 6 & 400 & 147 & 553 \\
parto/curetagem & 5 & 218 & 56 & 279 \\
Após o parto & - & 8 & 4 & 12 \\
Não realizado & 24 & 1391 & 211 & 1626 \\
\hline Total & & & &
\end{tabular}

Legenda: (Ign) ignorado; (-) dado numérico igual a 0.

Fonte: Nunes IR, et al., 2020. Baseado em: Ministério da Saúde/SVS

- Sistema de Informação de Agravos de Notificação - Sinan Net, 2018.

No decorrer do período analisado, somente $30,56 \%$ dos parceiros (497 indivíduos) realizaram tratamento concomitantemente às gestantes infectadas. Infelizmente, constata-se que ao decorrer dos anos esse número aumenta gradativamente, verificando-se um percentual de $63 \%$ de parceiros em tratamento durante o ano de 2017.

Quanto à classificação final dos casos, nota-se que grande parte, 1340 casos $(82,51 \%)$ foi considerada sífilis congênita recente e ressalta-se a grande quantidade de casos em que essa informação foi ignorada ou deixada em branco, 172 registros (Gráfico 2).

Gráfico 2 - Classificação final dos casos confirmados de sífilis congênita notificados no Piauí entre 2013 a 2017.

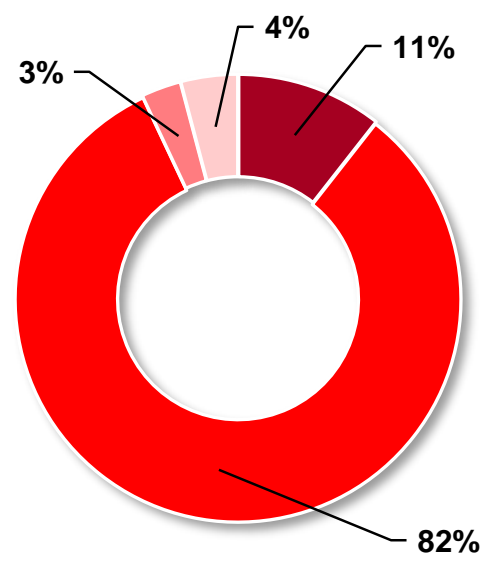

- Ignorado/Branco - Sífilis Congênita Recente " Natimorto/Aborto por Sífilis Descartado

Fonte: Nunes IR, et al., 2020. Baseado em: Ministério da Saúde/SVS - Sistema de Informação de Agravos de Notificação - Sinan Net, 2018.

No que se refere à mortalidade infantil, entre os anos de 2013 a 2017 ocorreram 35 (2,15\%) óbitos por SC no estado (Gráfico 3). O coeficiente de mortalidade mostrou tendência de crescimento, pois em 2013 foram 4,30 casos por 10.000 nascidos vivos, elevando-se para 20,30 casos $/ 10.000$ nascidos vivos em 2015 e caindo para 14,90 casos em 2016. Entretanto, não foi possível calcular este coeficiente para o ano de 2017 devido a indisponibilidade de dados no SINASC para esse ano. 
Gráfico 3 - Coeficiente de mortalidade notificados no Piauí entre 2013 a 2017.

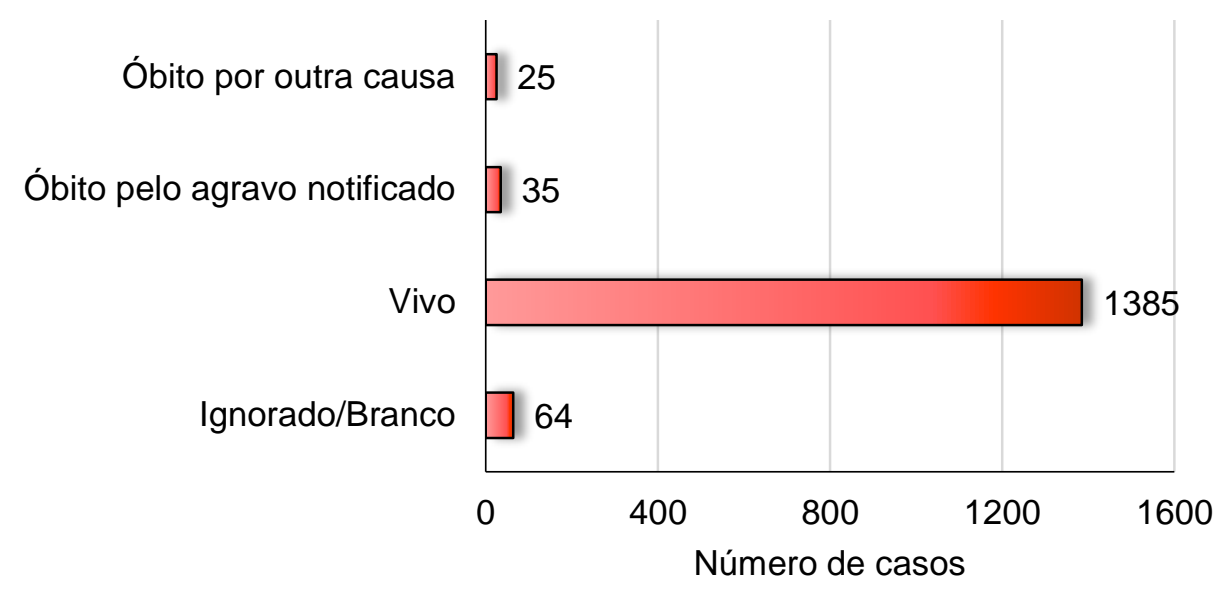

Fonte: Nunes IR, et al., 2020. Baseado em: Ministério da Saúde/SVS - Sistema de Informação de Agravos de Notificação - Sinan Net, 2018.

\section{DISCUSSÃO}

O Brasil ficou signatário da Resolução CE116/14 da Organização Pan-Americana da Saúde (OPAS), no qual em 1995 assumiu o acordo de elidir a incidência de sífilis congênita até o ano 2000, estabelecendo um programa de controle da enfermidade fundamentando na pratica da assistência pré-natal e na análise e método apropriado dos casos de sífilis gestacional, de maneira a proteger a transmissão vertical da doença (LIMA MG, et al., 2013)

O aumento das ocorrências de SC evidenciado no período de 2013 à 2017 demonstrou-se que mesmo com os meios de prevenções disponíveis para população, ainda não se obteve uma estabilidade da doença. Ao contrário, no ano de 2015 denotou-se uma maior elevação no total de casos observados, com isso a SC permanece como uma das relevantes preocupações da saúde pública no Brasil. No que se refere a disseminação espacial dos casos ressalta-se a ocorrência da doença em vários municípios Piauienses, onde destacou-se o município de Teresina, seguido por Parnaíba e Picos. Já em vista aos resultados pertinentes às características maternas, houve predominância de casos nas gestantes com escolaridade incompleta do ensino fundamental.

O fato excessivo de sífilis congênita, apesar de haver providencias para sua prevenção, mostra que encontram-se muitos erros nos sistemas de saúde, principalmente no pré-natal, o que causa inúmeros sinais desfavoráveis para a saúde, como o aumento das taxas de óbito por aborto, natimorto ou óbito neonatal precoce, que chegam a cera de 25 a $35 \%$ dos casos (GONÇALVES J, et al., 2011). Nosso trabalho, contudo, validou que $95,26 \%$ foram diagnosticados em bebês com até seis dias de vida, 1,84\% em bebês com 7 a 27 dias de nascimento e 2,83\% em crianças com um ano de idade ( 12 a 23 meses).

No decorrer do período analisado, obteve-se um baixo número de parceiros que realizaram o tratamento concomitantemente às gestantes infectadas. Infelizmente, constatou-se que ao decorrer dos anos esse número aumentou gradativamente. Contudo, vale ressaltar que a inserção dos companheiros nas atividades educativas durante o pré-natal é um requisito fundamental para a obtenção de um provável controle da SC.

Logo, é sabido que no período gestacional a receptividade e aceitação dos pais, é essencial para impossibilitar a reincidência da doença. Entretanto $30,56 \%$ dos parceiros realizaram tratamento concomitantemente às gestantes infectadas, constata-se que ao decorrer dos anos esse número aumenta gradativamente. Á visto disso, segundo Costa CC, et al. (2013), foi restrito o domínio da sífilis na gestação, uma vez que vários parceiros não realizam tratamento adequado. A situação comprova que as gestantes com sífilis sentem a inaptidão do tratamento, o contágio e a transmissão vertical. De acordo com Lima MG, et al. (2013) a falta de tratamento apropriado amplia o perigo de contágio da sífilis da mãe para o embrião. 
Estudos mostram a intervenção das diferenças sociáveis no ataque da SC. Logo enfatiza que não foram observadas diferenças de distribuição relevantes conforme o sexo. A escolaridade materna, evidenciou-se que foi o maior número $29,12 \%$, não concluiu as séries básicas do ensino fundamental ( $5^{\underline{a}}$ à $8^{\text {a }}$ série), seguida de $17,80 \%$ com o ensino médio incompleto, iniciaram o pré-natal tardio e, realizaram número inadequado de consultas pré-natal (DOMINGUES RMSM, et al., 2013; LIMA MG, et al., 2013). Ao que se refere à mortalidade infantil, o coeficiente de mortalidade mostrou tendência de crescimento, principalmente no ano de 2015 , tendo um declínio de forma gradativa no ano de 2016. Contudo, no que tange a essa categoria, não se realizou o cálculo de coeficiente para o ano de 2017, justificado pela indisponibilidade de dados no SINASC.

\section{CONCLUSÃO}

O estudo mostrou o predomínio da SC caracterizando o perfil epidemiológico e os fatores associados à transmissão no Piauí, onde percebe-se que a SC possui uma grande relevância dentro do cenário da saúde, tanto ao nível de Brasil quanto ao nível do estado do Piauí. Este trabalho nos mostra que a SC ainda está fora de controle no estado do Piauí, vista que o quantitativo de casos ainda é elevado. Ao modo que este cenário traz diversas motivações para se discutir ainda mais sobre a temática, e demonstra um ponto pertinente, que é a importância do tratamento de ambos no período gestacional ou após o nascimento, para evitar a reincidência da doença. Objetivando dessa forma levantar questionamentos inerentes para possíveis resolutividades da causa. Contudo, acredita-se que mesmo com algumas limitações, o objetivo foi alcançado, conseguindo contemplar com êxito o esperado pelo trabalho. Todavia, é com assertividade que este trabalho irá facilitar futuras propostas que visem conhecer e estabelecer o predomínio da sífilis congênita de forma que caracterize o perfil epidemiológico e fatores associados à transmissão no Piauí.

\section{REFERÊNCIAS}

1. ALMEIDA PD, et al. Análise epidemiológica da sífilis congênita no Piauí. Revista Interdisciplinar, 2015; 8(1): 62-70.

2. ARAÚJO CL, et al. Incidência da sífilis congênita no Brasil e sua relação com a Estratégia Saúde da Família. Revista de Saúde Pública, 2012; 46, 479-486.

3. BRASIL. Secretaria de Vigilância em Saúde, Departamento de DST, Aids e Hepatites Virais. Boletim Epidemiológico - Sífilis. Brasília: Ministério da Saúde; 2015.

4. BRASIL. Ministério da Saúde. Diretrizes para controle da Sífilis Congênita. Secretaria Nacional de Vigilância em Saúde. Brasília, DF, 2006.

5. CASAGRANDE A, et al. Evolução da qualidade das informações das declarações de óbito com menções de sífilis congênita nos óbitos perinatais no Brasil. Cadernos Saúde Coletiva, 2017; 25(3).

6. CARVALHO IS, BRITO RS. Sífilis congênita no Rio Grande do Norte: estudo descritivo do período 2007-2010. Epidemiol Serv Saúde. 2014; 23(2): 287-94.

7. COSTA CC, et al. Sífilis congênita no Ceará: análise epidemiológica de uma década. Revista da Escola de Enfermagem da USP, 2013; 47(1): 152-159.

8. DOMINGUES RMSM, et al. Sífilis congênita: evento sentinela da qualidade da assistência pré-natal. Revista de Saúde Pública, 2013; 47: 147-157.

9. FIGUEIREDO MSN, et al. Percepção de enfermeiros sobre a adesão ao tratamento dos parceiros de gestantes com sífilis. Revista da Rede de Enfermagem do Nordeste, 2015; 16(3).

10. GONÇALVES J, et al. Perfil epidemiológico dos casos de sífilis congênita de um hospital universitário - 2004 a 2008. Revista Brasileira de Pesquisa em Saúde/Brazilian Journal of Health Research, Vitória, 2011; 13(2): 49-55.

11. GONÇALVES HC, et al. Incidência de sífilis congênita no estado de Santa Catarina no ano de 2012. Arquivos Catarinenses de Medicina, 2017; 46(2): 15-25.

12. HOLANDA MTCGD, et al. Perfil epidemiológico da sífilis congênita no Município do Natal, Rio Grande do Norte-2004 a 2007. Epidemiologia e Serviços de Saúde, 2011; 20(2): 203-212.

13. LIMA MG, et al. Incidência e fatores de risco para sífilis congênita em Belo Horizonte, Minas Gerais, 2001 2008. Ciência \& Saúde Coletiva, 2013; 18: 499-506.

14. SOUZA BC, SANTANA LS. As consequências da sífilis congênita no binômio materno-fetal: um estudo de revisão. Interfaces Científicas-Saúde e Ambiente, Aracaju, 2013; 1(3): 59-67.

15. VASCONCELOS MIO, et al. Sífilis na gestação: estratégias e desafios dos enfermeiros da atenção básica para o tratamento simultâneo do casal. Revista Brasileira em Promoção da Saúde, 2017; 29: 85-92. 